\title{
Hydrodynamic study of a microwave plasma torch
}

\author{
K. Gadonna ${ }^{1}$, O. Leroy ${ }^{1}$, T. Silva ${ }^{2}$, P. Leprince ${ }^{1}$, C. Boisse-Laporte ${ }^{1}$ and L.L. Alves ${ }^{2}$ a \\ 1 Laboratoire de Physique des Gaz et des Plasmas, UMR CNRS/UPS 8578, Orsay, France \\ 2 Instituto de Plasmas e Fusão Nuclear, Instituto Superior Técnico, Universidade Técnica de Lisboa, Lisboa, Portugal
}

Received: date / Revised version: date

\begin{abstract}
A hydrodynamic model was developed to simulate the flow and the heat transfer with the gas/plasma system produced by a microwave-driven $(500-900 \mathrm{~W}$ at $2.45 \mathrm{GHz})$ axial injection torch, running in atmospheric pressure helium at $3-9 \mathrm{~L} \mathrm{~min}^{-1}$ input gas flows. The model solves the NavierStokes' equations, including the effect of the plasma upon the momentum and the energy balance, in order to obtain the spatial distributions of the gas velocity and temperature. The model predicts average gas temperatures of $2500-3500 \mathrm{~K}$, in the same range of those obtained by optical measurements. Simulations show that the plasma influences the gas flow path and temperature, promoting an efficient power transfer.
\end{abstract}

\section{Introduction}

Among the different types of microwave plasma torches, the axial injection torch (TIA for Torche à Injection Axiale in French) [1] has been used to create chemically active species for gas analysis, surface processing, and gaseous waste treatments $[2,3]$, or simply to achieve a volumetric gas heating. Here, helium is injected at atmospheric pressure into a TIA (with flow rates of a few $\mathrm{L} / \mathrm{min}$ ), producing a high luminosity, high density plasma [4] $(3 \mathrm{~cm}$ length and $1 \mathrm{~mm}$ radius) at the nozzle's exit. The nozzle acts also as a microwave coaxial antenna, delivering a $500-900 \mathrm{~W}$ power at $2.45 \mathrm{GHz}$ frequency.

The present study focus on the hydrodynamic description of a TIA, by solving the Navier-Stokes' equations for the gas/plasma system including the gas energy balance equation, in order to analyse the influence of the plasma and of the input gas flow on the spatial distributions of the gas velocity and temperature. The plasma and the wall temperature conditions required to run the model are taken from experiment.

\section{Model formulation and solution}

\subsection{Navier-Stokes' equations}

The hydrodynamic characteristics of this problem have been discussed elsewhere [5] for a closed reactor configuration. The distribution of velocity $\boldsymbol{v}$ and temperature $T_{g}$ with the heavy particles, in the presence of a plasma heating source, is calculated by solving the corresponding mass, momentum, and energy balance equations for neutral helium (in the present conditions, the neutral gas

\footnotetext{
${ }^{a}$ e-mail: llalves@ist.utl.pt
}

density is about $10^{18}-10^{19} \mathrm{~cm}^{-3}$, thus much larger than the plasma ion density $\sim 10^{14} \mathrm{~cm}^{-3}$ )

$$
\begin{gathered}
\boldsymbol{\nabla} \cdot(\rho \boldsymbol{v})=0 \\
\rho(\boldsymbol{v} \cdot \boldsymbol{\nabla}) \boldsymbol{v}+\boldsymbol{\nabla} p+\boldsymbol{\nabla} \cdot \boldsymbol{\tau}=e n_{i} \boldsymbol{E}_{\mathbf{d c}} \\
\rho C_{v}(\boldsymbol{v} \cdot \boldsymbol{\nabla}) T_{g}-\boldsymbol{\nabla} \cdot\left(\lambda_{g} \boldsymbol{\nabla} T_{g}\right)+p \boldsymbol{\nabla} \cdot \boldsymbol{v} \\
=3 \frac{m_{e}}{M_{\mathrm{He}}} n_{e} \nu_{e n} k_{B}\left(T_{e}-T_{g}\right)+e \boldsymbol{\Gamma}_{\boldsymbol{i}} \cdot \boldsymbol{E}_{\mathbf{d c}}
\end{gathered}
$$

where $p=\left(\rho / M_{\mathrm{He}}\right) k_{B} T_{g}$ is the gas pressure (with $\rho$ the mass density, $M_{\mathrm{He}}$ the mass of an helium atom, and $k_{B}$ the Boltzmann's constant); $\tau$ is the viscosity tensor given by $\boldsymbol{\tau}=-\eta\left[\boldsymbol{\nabla} \boldsymbol{v}+(\boldsymbol{\nabla} \boldsymbol{v})^{T}-(2 / 3) \boldsymbol{\nabla} \cdot \boldsymbol{v} \boldsymbol{I}\right][$ with $\eta=(5 / 16 \pi)$ $\left(\pi M_{\mathrm{He}} k_{B} T_{g}\right)^{1 / 2} /\left(\sigma^{2} \Omega\left(T_{g}\right)\right)$, where $\sigma$ is the collision diameter and $\Omega$ is the collision integral [6], and $\boldsymbol{I}$ the identity tensor]; $C_{v}=(3 / 2)\left(k_{B} / M_{\mathrm{He}}\right)$ is the gas heat capacity at constant volume; $\lambda_{g}=(15 / 4) \eta\left(k_{B} / M_{\mathrm{He}}\right)$ is the gas thermal conductivity $[6] ; \nu_{e n}\left(\mathrm{~s}^{-1}\right)=6.8 \times 10^{-8} n_{g}$ $\left(\mathrm{cm}^{-3}\right)$ is the electron-neutral collision frequency [7] (with $n_{g}=\rho / M_{\mathrm{He}}$ the gas density); $m_{e}, n_{e}$ and $T_{e}$ are the electron mass, density and temperature, respectively; and $\boldsymbol{E}_{\mathbf{d c}}, n_{i}$ and $\boldsymbol{\Gamma}_{\boldsymbol{i}}$ are the space-charge field, the ion density and the ion flux, respectively, calculated here for ambipolar conditions

$$
\begin{gathered}
\boldsymbol{E}_{\mathrm{dc}} \simeq-\frac{k_{B} T_{e}}{e} \frac{\boldsymbol{\nabla} n_{e}}{n_{e}} \\
n_{i} \simeq n_{e} \\
\boldsymbol{\Gamma}_{\boldsymbol{i}} \simeq-D_{a} \boldsymbol{\nabla} n_{e}
\end{gathered}
$$

with $D_{a} \simeq\left(k_{B} T_{e} / e\right) \mu_{i}$ the ambipolar diffusion coefficient and $\mu_{i} n_{g}=4.49 \times 10^{20} \mathrm{~V}^{-1} \mathrm{~cm}^{-1} \mathrm{~s}^{-1}$ the reduced ion mobility [7] (for molecular ions $\mathrm{He}_{2}^{+}$, the sole ion species considered under atmospheric pressure conditions).

Equations (1b)-(1c) (the latter deduced from $a b$ initio calculations, which correct the formulation adopted by 
other authors $[8,9]$ when writing the balance of the internal energy with the system) update our previous hydrodynamic description of the problem, by considering the ion drag-force $\left(e n_{i} \boldsymbol{E}_{\mathbf{d c}}\right)$ and Joule heating $\left(e \boldsymbol{\Gamma}_{\boldsymbol{i}} \cdot \boldsymbol{E}_{\mathbf{d c}}\right)$ supplementary terms to account for the plasma influence on the gas transport and heating. The latter is achieved mainly through elastic electron-neutral collisions, described by the first term on the right-hand side of equation (1c).

\subsection{Work conditions and numerical solution}

The axis-symmetric setup under study (see figure 1-left) consists of a nozzle $(2 \mathrm{~mm}$ diameter for the gas injection channel) located inside a dielectric tube $(57 \mathrm{~cm}$ length and $5 \mathrm{~cm}$ diameter). The input gas flow is of $3-9 \mathrm{~L} \mathrm{~min}^{-1}$ (yielding input axial average velocities of $\left\langle v_{\mathrm{in}_{z}}\right\rangle=16-$ $50 \mathrm{~m} \mathrm{~s}^{-1}$ ), which ensures subsonic and non-turbulent flow conditions (the Mach number and the Reynolds' number are $\mathrm{M}<0.3$ and $\operatorname{Re}<2000$, respectively). Here the

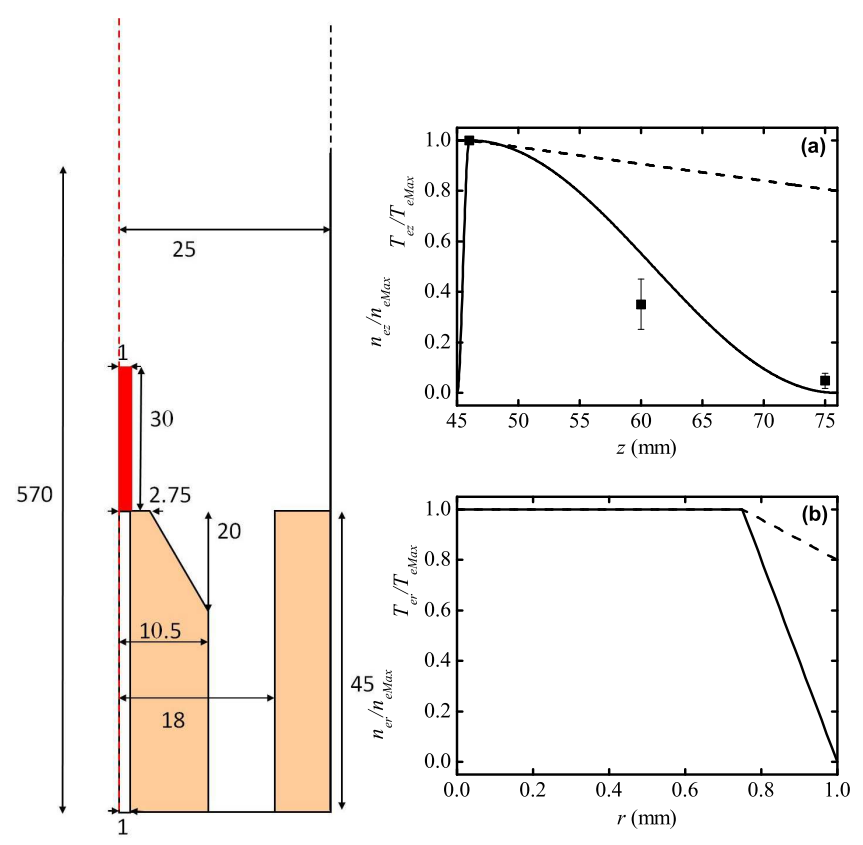

Fig. 1. Left: axis-symmetric 2D computational domain adopted in the simulations (dimensions are in $\mathrm{mm}$ and the diagram is not to scale). The plasma (in red) is located at the nozzle's end (starting at position $z=45 \mathrm{~mm}$ ). The orange region represents the coaxial antenna. Right: radial (a) and axial (b) profiles for the electron density (solid curves) and temperature (dashed curves). The points are OES measurements of $n_{e}$.

plasma is defined by imposing the radial and axial profiles $X_{e}(r, z)=X_{e M A X} X_{e r}(r) X_{e z}(z)$ (with $X \equiv n, T$ ) shown in figures 1-right(a) and (b). Note that the axial profile of $n_{e}$ (a combination of co-sinus functions) is chosen according to optical spectroscopy measurements (OES) of the Stark broadening of the $\mathrm{H}_{\alpha}$ and the $\mathrm{H}_{\beta}$ atomic lines.
These measurements show that the maximum of the light emitted by the plasma is located $\sim 1 \mathrm{~mm}$ away from the nozzle, suggesting the formation of a hollow axial profile for $n_{e}$ [see figure 1-right(a)], due to a dragging effect. Typical values for the electron density and temperature maxima (the latter defined according to [10]) are $n_{e M A X} \sim 3 \times 10^{14} \mathrm{~cm}^{-3}$ and $T_{e M A X} \sim 2 \times 10^{4} \mathrm{~K}$.

The boundary conditions are the following. At the axis $(r=0)$, axis-symmetry conditions are used. At the walls (of the coaxial antenna and the dielectric), we impose $\boldsymbol{v}=0$ (corresponding to a no-slip condition), the $T_{g}$ values measured with a thermal probe (at the dielectric), and $T_{g}=390 \mathrm{~K}$ (at the metal). At the input opening $(z=0)$ we set the gas velocity according to $v_{z}(r)=$ $2\left\langle v_{\mathrm{in}_{z}}\right\rangle\left[1-(r / R)^{2}\right]$ ( $R$ is the nozzle's radius); at the output opening we set the pressure $p=p_{\text {atm }}$ and the gas temperature $T_{g}=290 \mathrm{~K}$.

Simulations use the commercial software COMSOL Multiphysics ${ }^{\circledR}$ [11] adopting the solver PARDISO [12]. Meshing is performed automatically using triangular features; the convergence criterion imposes relative errors, between consecutive calculations, smaller than $10^{-6}$.

\section{Results and discussion}

\subsection{Influence of the plasma}

Figures 2(a)-(b) plot, respectively, the axial profile (at $r=0$ ) within the plasma region and the radial profile (at $z=15 \mathrm{~mm}$, corresponding to half of the plasma height), up to $10 \mathrm{~mm}$ outside the plasma, of the gas temperature calculated for an input gas flow of $5 \mathrm{~L} \mathrm{~min}{ }^{-1}$, at various $n_{e M A X}$ and $T_{e M A X}$ values. Notice that $T_{g}$ is very sensitive to changes in the electron energy-density, exhibiting an increase with both $n_{e M A X}$ and $T_{e M A X}$ due to the enhancement in the plasma-to-gas collisional power transfer.

To allow for a comparison with experimental results, figure 3 shows the axial profile of the calculated radially average gas temperature $\left\langle T_{g}\right\rangle_{r}$ and of the measured rotational temperature, obtained by fitting the ro-vibrational spectra emitted by the second positive system of $\mathrm{N}_{2}$, using the SPECAIR software [13]. These diagnostics are performed perpendicularly to the discharge axis, by collecting all the light emitted by the plasma at given $z$ positions, for different microwave powers. One observes that the calculated gas temperatures have the same order of magnitude than the measured ones. The differences in the axial variation of $T_{g}$, predicted by simulations and deduced by measurements, might evidence the fact that we didn't adopt self-consistent plasma profiles in the present calculations, but can also introduce a discussion about the experimental results (beyond the mere observation that measurements have a significant uncertainty, confirmed by the errors bars depicted in figure 3 ). In fact, the nitrogen used in the measurements is dragged into the plasma-jet from the air envelope which surrounds it, meaning that probably the experimental gas temperature doesn't accurately account for the plasma-to-gas heat transfer occurring near the discharge axis. 

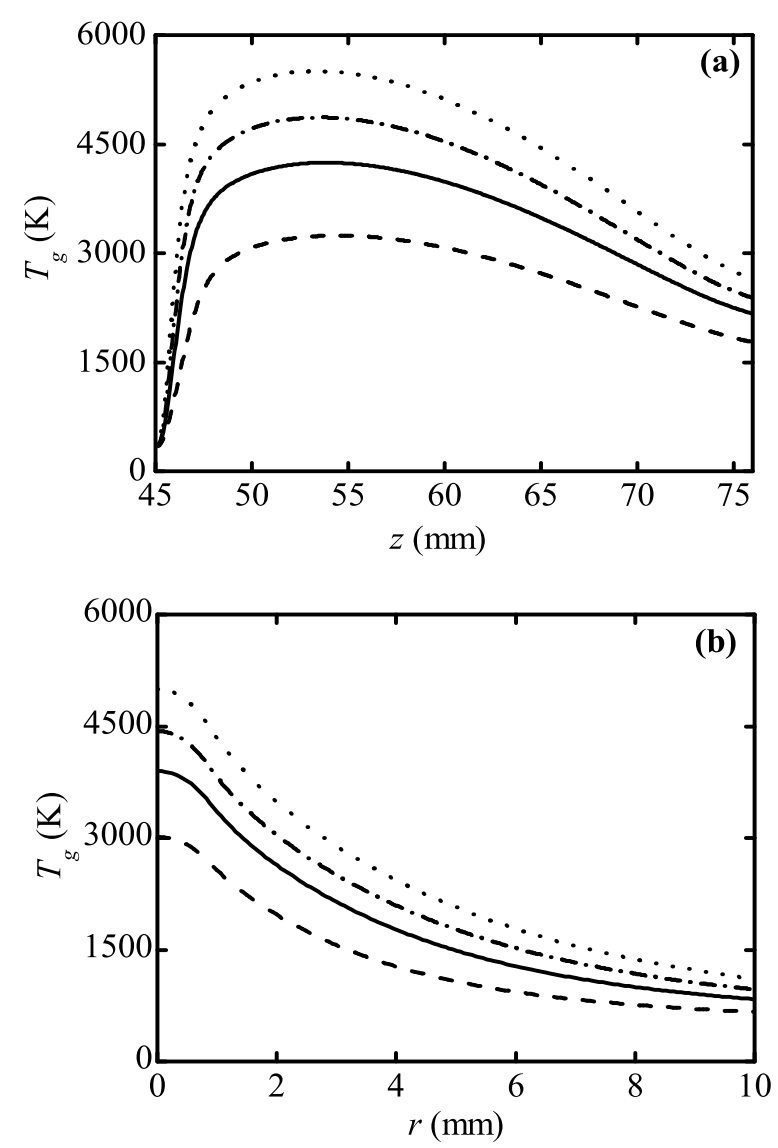

Fig. 2. (a) Axial profile (at $r=0$ ) and (b) radial profile (at $z=15 \mathrm{~mm}$ ) of the gas temperature in the plasma nearby region, calculated at $5 \mathrm{~L} \mathrm{~min}^{-1}$ input gas flow, for (i) $T_{e M A X}=$ $2 \times 10^{4} \mathrm{~K}$ and the following $n_{e M A X}$ values (in $10^{14} \mathrm{~cm}^{-3}$ ): 1.5 (dashed curves), 3 (solid), 6 (dotted); (ii) $T_{e M A X}=2.7 \times 10^{4} \mathrm{~K}$ and $n_{e M A X}=3 \times 10^{14} \mathrm{~cm}^{-3}$ (dashed-dotted).

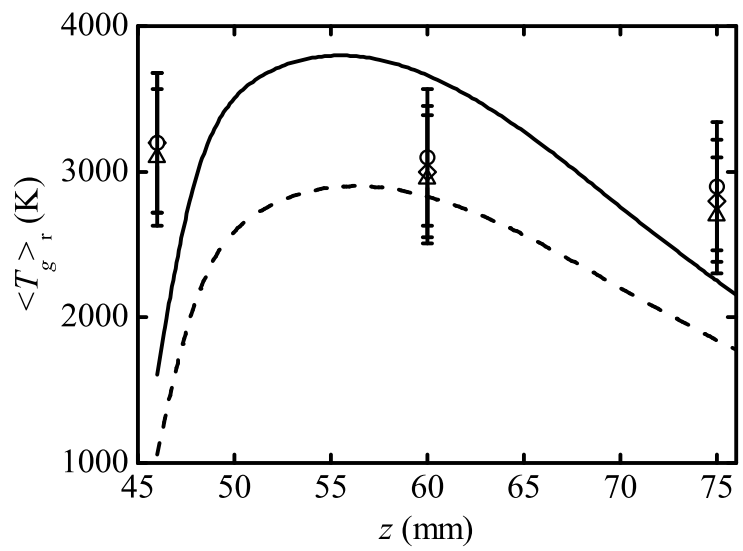

Fig. 3. Axial profile of $\left\langle T_{g}\right\rangle_{r}$, for the gas temperature calculated in the same conditions as in figure 2 (curves) and for the measured rotational temperature (points), obtained at $5 \mathrm{~L} \mathrm{~min}{ }^{-1}$ input gas flow for the following microwave powers (in W): 500 (triangles), 600 (diamonds), 700 (circles).
Figure 4 plots, within the plasma region, the axial profile (at $r=0$ ) of the modulus of the gas velocity $|\boldsymbol{v}|$, calculated for the same conditions as before. The results in this figure show that the plasma influences also the gas flow path, acting as an obstacle responsible for a velocity peak whose intensity increases with the electron energy-density (i.e. with both $n_{e M A X}$ and $T_{e M A X}$ ). For $n_{e M A X}=3 \times 10^{14} \mathrm{~cm}^{-3}$ and $T_{e M A X}=2 \times 10^{4} \mathrm{~K}$, figure 4 shows results calculated with and without the supplementary ion term in equation (1b). One can observe the influence of the negative ion drag-force at the beginning of the plasma [for $45 \leq z(\mathrm{~mm}) \leq 46$ where the electron density is rapidly growing, see figure 1-right(a)], which extends throughout the entire plasma region to induce a more rapid decrease in the axial profile of $|\boldsymbol{v}|$.

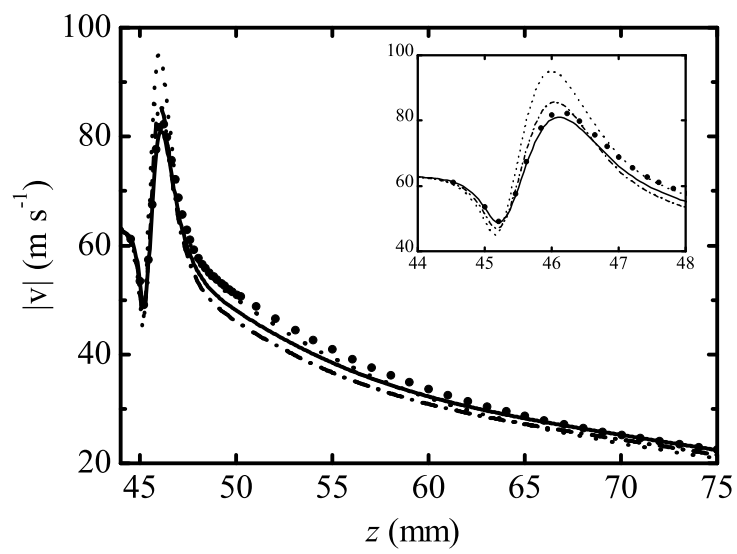

Fig. 4. Axial profile (at $r=0$, within the plasma region) of the modulus of the gas velocity, calculated at $5 \mathrm{~L} \mathrm{~min}^{-1}$ input gas flow, for (i) $T_{e M A X}=2 \times 10^{4} \mathrm{~K}$ and the following $n_{\text {eMAX }}$ values (in $10^{14} \mathrm{~cm}^{-3}$ ): 3 (solid, with ion terms; circles, without ion terms), 6 (dotted); (ii) $T_{e M A X}=2.7 \times 10^{4} \mathrm{~K}$ and $n_{e M A X}=3 \times 10^{14} \mathrm{~cm}^{-3}$ (dashed-dotted). The insert is a zoom over the beginning of the plasma region.

\subsection{Influence of the input gas flow}

Figure 5 shows the influence of the input flow on the gas temperature profile, inside and outside the plasma region. Under flow conditions, the gas temperature features a hollow spatial distribution near the nozzle's tip, whose depth becomes larger for longer energy relaxation lengths, associated with the effectiveness of the plasma-to-gas heat transfer. Figure 5 shows that an increase in the input gas flow leads to longer relaxation lengths in the axial direction for $T_{g}$, yielding a decrease in its maximum (in the plasma region) and a simultaneous increase in its tail (outside the plasma). However, for the fixed $n_{e}$ and $T_{e}$ profiles considered, the maximum of the gas temperature profile is located always around $z=54 \mathrm{~mm}$ in the axial direction [see figure $5(\mathrm{a})$ ], which in any case is after the maximum of the electron energy-density $n_{e} T_{e}$ [see figure 1-right(a)]. 

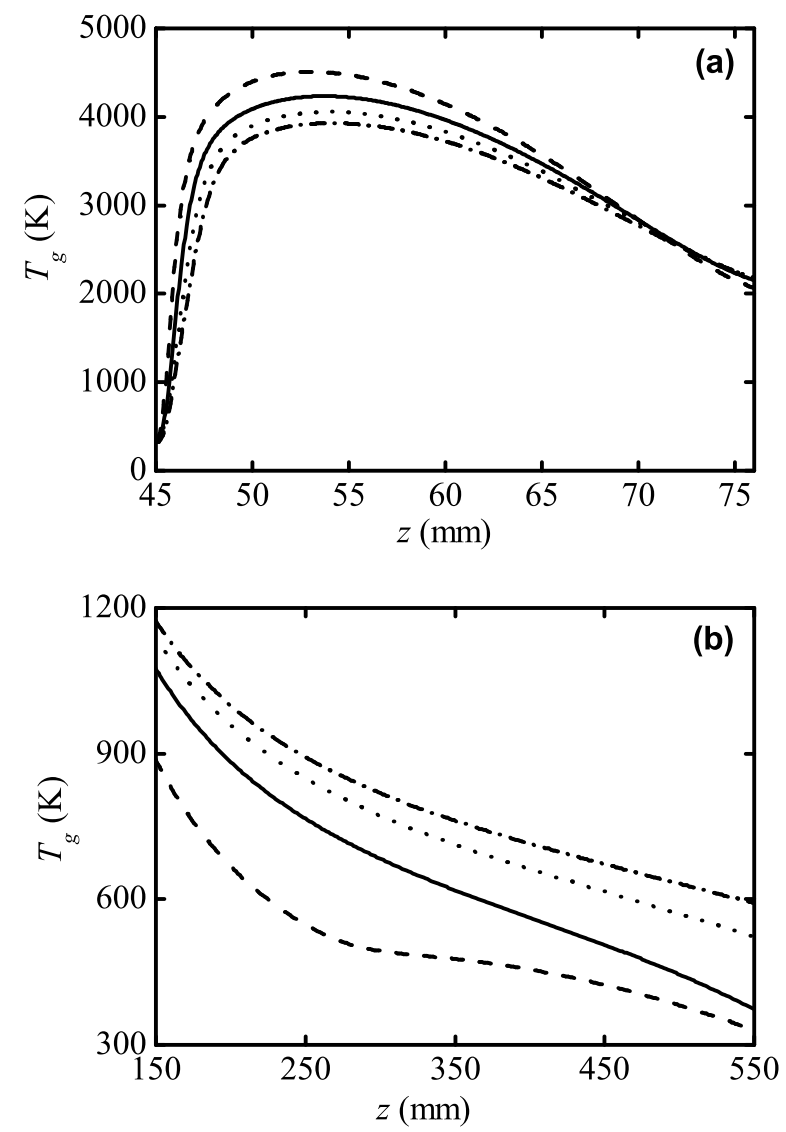

Fig. 5. Axial profile (at $r=0$ ) of the gas temperature inside the plasma region (a) and outside the plasma region (b), calculated for $n_{e M A X}=3 \times 10^{14} \mathrm{~cm}^{-3}, T_{e M A X}=2 \times 10^{4} \mathrm{~K}$ and the following input gas flows (in $\mathrm{L} \min ^{-1}$ ): 3 (dashed curves), 5 (solid), 7 (dotted), 9 (dashed-dotted).

This explains the more effective plasma-to-gas heat transfer occurring at lower input gas flows.

Experimentally, we observe the plasma length to increase with the input gas flow, evidencing the important role played by the residence time of species. In the present simulations, with imposed electron density and temperature profiles, the influence of the plasma length cannot be evaluated self-consistently. However, numerical tests show that an increase in the plasma length (at constant maximum electron density) leads to higher $T_{g}$ maxima, thus reducing the effect observed in figure $5(\mathrm{a})$, also favouring the gas heating outside the plasma region.

\section{Final remarks}

The development of a hydrodynamic model for a microwave-driven TIA, operating in atmospheric pressure helium in an open reactor configuration, has allowed to describe the influence of the plasma (using prescribed profiles for the electron density and temperature with maximum values of $\sim 3 \times 10^{14} \mathrm{~cm}^{-3}$ and $\sim 2 \times 10^{4} \mathrm{~K}$, respectively) and of the input gas flow $\left(3-9 \mathrm{~L} \mathrm{~min}^{-1}\right)$ on the gas velocity and temperature distributions. The model solves the Navier-Stokes' equations, including the effect of the plasma in the momentum and the energy balance equations, where some supplementary terms were considered to account for the drag-force and the Joule heating due to the ions.

The model predicted average gas temperature values of $2500-3500 \mathrm{~K}$, in the same range of those obtained by OES diagnostics. Simulations showed also that the plasma changes the path of gas flow and that there is an efficient plasma-to-gas power transfer, which demonstrates the influence of the electron energy-density on the gas heating via elastic electron-neutral collisions. Moreover, an increase in the input gas flow was found responsible for (i) longer relaxation lengths in the axial direction for the gas temperature, and (ii) a decrease in its maximum value.

The present work is part of a more extensive research program for the self-consistent modelling of the TIA. Work is in progress to develop a plasma model, describing the two-dimensional drift-diffusion transport of charged particles in the system. This model will provide self-consistent information about the plasma (in terms of its size and of the charged particle density and temperature), through its coupling with the hydrodynamic model, improving the description of the gas flow and heat transfer.

\section{Acknowledgements}

Work partially supported by the Portuguese Foundation for Science and Technology (FCT) and the Portuguese Ministry of Science, Technology and Higher Education (MCTES) (Project PTDC/FIS/65924/2006).

\section{References}

1. M. Moisan, G. Sauvé, Z. Zakrzewski and J. Hubert, Plasma Sources Sci. Technol. 3 (1994) 584

2. C. Tendero, C. Tixier, P. Tristant, J. Desmaison and P. Leprince, Spectrochimica Acta B 61 (2006) 2

3. S.J. Rubio, M.C. Quintero, A. Rodero and J.M. Fernandez Rodriguez, Journal of Hazardous Materials 148 (2007) 419

4. A. Ricard, L. St-Onge, H. Malvos, A. Gicquel, J. Hubert and M. Moisan, J. Phys. III 5 (1995) 1269

5. L.L. Alves, R. Alvarez, L. Marques, J. Rubio, A. Rodero and M.C. Quintero, Eur. Phys. J. Appl. Phys. 46 (2009) 21001

6. J.O. Hirschfelder, C.F. Curtiss, R.B. Bird, Molecular Theory of Gases and Liquids (John Wiley, 1964)

7. L.L. Alves and C.M. Ferreira, J. Phys. D: Appl. Phys. 24 (1991) 581

8. J. Henriques, E. Tatarova and C.M. Ferreira, J. Appl. Phys. 109 (2011) 023301

9. Y. Kabouzi, D.B. Graves, E. Castaños-Martínez and M. Moisan, Phys. Rev. 75 (2007) 016402

10. J. Torres, O. Carabaño, M. Fernández, S. Rubio, R. Álvarez, A. Rodero, C. Lao, M.C. Quintero, A. Gamero and A. Sola, J. Phys. Conference Series 44 (2006) 70

11. http://www.comsol.com/

12. http://www.pardiso-project.org/

13. http://www.specair-radiation.net/ 